\title{
LA NATURE-ARTEFACT : ENTRE ANTHROPISATION ET ARTIALISATION, L'EXPÉRIENCE DU SYSTĖME GTP (GÉOSYSTĖME-TERRITOIRE-PAYSAGE)
}

\author{
Claude Bertrand et Georges Bertrand
}

Armand Colin | « L'Information géographique »

2014/3 Vol. 78 | pages 10 à 25

ISSN 0020-0093

ISBN 9782200929473

Article disponible en ligne à l'adresse :

https://www.cairn.info/revue-l-information-geographique-2014-3-page-10.htm

Distribution électronique Cairn.info pour Armand Colin.

(C) Armand Colin. Tous droits réservés pour tous pays.

La reproduction ou représentation de cet article, notamment par photocopie, n'est autorisée que dans les limites des conditions générales d'utilisation du site ou, le cas échéant, des conditions générales de la licence souscrite par votre établissement. Toute autre reproduction ou représentation, en tout ou partie, sous quelque forme et de quelque manière que ce soit, est interdite sauf accord préalable et écrit de l'éditeur, en dehors des cas prévus par la législation en vigueur en France. Il est précisé que son stockage dans une base de données est également interdit. 


\title{
La nature-artefact : entre anthropisation et artialisation, l'expérience du système GTP (Géosystème-Territoire-Paysage)
}

\author{
Par Claude et Georges Bertrand
}

Claude et Georges Bertrand, Géode, Géographie de l'environnement, UMR CNRS, Université de Toulouse-Le Mirail - gbertran@univ-tlse2.fr

« Depuis Descartes, de plus en plus de choses dépendent de nous. On est désormais dans une période ou nous dépendons de choses qui dépendent de nous ».

Michel Serres

Le concept oxymore de nature-artefact réalise une combinatoire entre artialisation au sens le plus large et anthropisation que le positivisme scientifique a séparées. Ainsi rassemblée, l'artificialisation apparaît comme le moteur essentiel de la crise environnementale. Elle est ici érigée en principe téléologique central du système GTP (geosysteme-territoire-paysage). Le GTP systémise, scénarise et mieux finalise, en prise directe avec une crise des territoires qui ne fait que commencer et dont la nature est une dimension incontournable.

\section{- Au seuil de l'Anthropocène...}

Nous entrons, dit-on, dans le nouvel âge de l'Anthropocène. Par la porte de service. Celle d'une crise environnementale jugée sans précédent, du moins dans la (très courte) histoire de l'humanité. Crise exceptionnelle, peut-être par sa profondeur et son irréversibilité, certainement par l'impact de l'anthropisation, rapide, violent et surtout inédit. Si l'« Homme » se place toujours au centre de l'Univers, l'anthropocentrisme est devenu abusif, pernicieux, absurde. La « Nature », du moins ce que nous désignons ainsi, en est réduite à jouer les utilités, comme ressource, et les futilités, comme décor. Tout en demeurant, puissance immanente, comme un deus ex machina, imprévisible, brutal, irréductible.

Notre rapport à la nature a changé : la nature change, nous changeons la nature, la nature nous change. L'artificialisation est devenue sinon la règle du moins le processus dominant, avec son cortège de dégradations et de crises vécues comme autant de catastrophes - mais aussi de beautés révélées. Vers un futur d'artifice pour l'humanité et la planète. 
Entrer dans l'Anthropocène constitue une rupture. Le concept de nature-artefact est un premier seuil à franchir entre environnement et paysage, entre culture et nature.

\section{" Le naturalisme est le passager clandestin de notre histoire "}

La célèbre phrase de Serge Moscovici date, déjà, des années 1968. Citée comme une évidence parmi d'autres évidences écologisantes, elle a suscité de nombreuses réserves, quelques contresens et beaucoup de controverses. Il est mal venu d'avoir une « nature » d'avance sur son temps. Un demi-siècle plus tard cette profession de foi sonne haut et clair dans un monde de plus en plus chaotique et artificialisé. Avec, quelque part, une nature équivoque, masquée sous un habillage (camouflage ?) environnemental anthropocentré et vaguement (hypocritement ?) humanitariste. Une clandestinité irréaliste, insupportable, dangereuse. L'en extraire suppose un biais d'intercession à contre-courant des pensées et méthodes dominantes. Avec quel(s) passeur(s) pour quel paradigme ?

\section{Incontournable, l'environnement n'est pas indépassable}

Depuis plus d'un demi-siècle, l'environnement s'est imposé comme instrument unique et univoque. Il a gagné une guerre planétaire mais il n'a pas remporté toutes les batailles et celles qui se profilent paraissent déjà compromises. Tel qu'il est aujourd'hui théorisé et pratiqué, il a révolutionné la représentation du monde au cours du $\mathrm{XX}^{\mathrm{e}}$ et au début du $\mathrm{XXI}^{\mathrm{e}}$ siècles. Il ne répond plus ni à l'état du monde ni à notre rapport au monde. Il reste toutefois incontournable, en particulier grâce à ses solides fondements écologiques et, depuis peu, économiques. Confronté à la crise globale, autant culturelle que matérielle, il est dépassé et incapable de s'adapter aux problématiques culturelles, politiques et sociales, en particulier celles des pays de Sud où il apparaît comme le nouvel avatar de l'impérialisme colonial.

\section{Sous les paysages... la nature}

L'environnement nous parle de la nature mais il ne nous en rapproche pas. Il lui manque cette familiarité et cette sensibilité que nous offre tout naturellement le paysage qu'il soit urbain ou rural. Toutefois, passer par les paysages pour débusquer une nature pourtant évidente n'est pas un chemin de simplification cartésienne. C'est plonger délibérément dans la complexité-diversité des sensations et des idées, par-delà les catégories philosophiques et les cloisonnements scientifiques. Une diagonale du flou. Avec, à mesure que l'on croit progresser, un empilement d'incertitudes et de contradictions :

- soit un paysage confondu avec la nature, soit un paysage d'artifice opposé à une nature « sauvage »; 
- soit un paysage amalgamé avec un environnement naturaliste, soit un paysage sensible, totalement artialisé et cadré dans des systèmes de représentation esthétisants et trop souvent ésotériques.

Quels paysages retenir? Aller au plus simple et au plus près de la vie. A priori, des paysages tangibles, réalités visibles, tactiles, audibles... dans leur diversité de villes et de campagnes, de rivières et de forêts, de vents et de nuages, tels qu'ils sont ressentis et vécus, dépouillés de toute logorrhée paysagistique dans leurs ordinaires quotidiens. La complexité paysagère viendra d'elle-même, chemin faisant, comme produit dérivé de l'artificialisation.

Proposer d'entrer dans l'Anthropocène, dans la longue sédimentation des temps géologiques, c'est, au-delà de l'histoire humaine, souligner l'immanence de la nature. C'est redonner à la nature une présence renforcée avec une visibilité nouvelle sous un nouvel éclairage... fût-il d'artifice.

- c'est d'abord le retour en force de la nature bio-physique avec son cortège d'instabilités matérielles (climatiques, tectoniques, biologiques) et les catastrophes humaines qui l'accompagnent et l'amplifient ;

- c'est aussi le retour non moins remarquable d'une nature sensible dans les manifestations, sociales et économiques et tout particulièrement dans le domaine des arts et de l'esthétique (cinéma, photographie, peinture, design, etc.).

\section{- Fuyante nature}

\section{"Chassez le naturel... »}

Sous cet intitulé « naturellement » provocateur nous avons, en 1989, interpellé les géographes français dans un billet d'humeur de l'Espace Géographique à propos de la déshérence de la géographie physique laminée entre une géographie sociale en pleine expansion et une géomorphologie qui brille de ses derniers feux. Cette mise en garde faisant suite à plusieurs publications de fond, dont les articles « Paysage et géographie physique globale » (significativement refusé par les Annales de géographie, publié dans la Revue géographique des Pyrénées et du Sud-Ouest en 1968) et « Une géographie contre-nature » (Hérodote, 1978). Des textes à la fois critiques et constructifs (proposition du concept de géosystème). Ils soulignent le manque d'intérêt de la discipline pour une nature devenue, en pleine euphorie des Trente Glorieuses, une question subsidiaire aussi bien dans les idéologies marxistes que libérales. Par ailleurs, la géographie française a été à peine effleurée par l'émergence de l'écologie scientifique des années 1960-1970. Autant d'occasions manquées pour une discipline qui n'assume plus sa position charnière entre les sciences de la société et les sciences de la nature. Fuyante géographie. 
Il fallait changer de discours et de méthode. C'est-à-dire de discipline et de public. L'occasion en a été la rédaction du premier chapitre de l' Histoire de la France rurale (Duby, 1975) : " Ouvrir une histoire des paysans par un tableau géographique des campagnes françaises c'est supposer le problème écologique résolu. C'est momifier un espace artificiellement stabilisé dans le temps et borné dans l'espace... c'est figer le mouvement de la nature et de l'histoire. »

Cette mise en perspective dynamique du « naturel des terroirs » dans l'évolution globale de la société et de la nature était destinée à rapprocher les histoires d'une nature considérée avec suspicion... et sans profondeur historique. L'écologie centrée sur le concept intégrateur d'écosystème en a été le vecteur essentiel.

Toutefois, si l'écologie a retrouvé l'unité du monde vivant, elle n'embrasse pas la totalité physique de la nature et ignore le paysage. Dans les années 1980, la « biogéographie des géographes » (G. Rougerie, G. Sautter, J.-C. Wieber, G. Bertrand) s'ouvre sur les «paysages vécus » et les «cadres de vie » mais elle demeure confidentielle dans sa discipline.

Au-delà de la prolifération des discours philosophiques sur « la nature de la nature », c'est surtout chez les ethnologues-anthropologues qu'il faut rechercher le rapport entre les sociétés et leurs natures. J. Barrau, C. Lévi-Strauss, G. Guille-Escuret, puis M. Godelier, P. Perez et Ph. Descola se posent directement la question dans toute son ambivalence.

C'est chez les paysagistes, surtout parmi les plus artialistes (A. Roger), et souvent à l'encontre de leurs démonstrations figées dans un esthétisme académique, qu'il faut chercher de nouveaux éclairages sur la nature à travers le prisme d'une culture éblouissante mais confinée dans la peinture, les parcs et les jardins. A contrario, les travaux de S. Briffaud sur les paysages pyrénéens (1980) montrent ce que leur « invention » doit, au cours des XVII ${ }^{\mathrm{e}}$ et XVIII ${ }^{\mathrm{e}}$ siècles, aux savants naturalistes (géologues, botanistes, géomètres-arpenteurs) bons connaisseurs pédestres de la nature pyrénéenne. Le naturalisme et le paysagisme ont une longue histoire commune et ne sont pas aussi étrangers l'un à l'autre qu'on veut bien le croire.

Des géographes se sont interrogés, tardivement et marginalement, sur la dimension naturaliste de leur discipline, soit à l'occasion de débats (Espace Géographique), soit d'études générales (A. Berque, M. Lussault) ou plus fréquemment de monographies de terrain (C. Blanc-Pamard, H. Rakoto Ramiarensoa, C. et G. Bertrand, P. Dérioz, L. Lelli, F. Le Du). La nature n'appartient pas à une discipline. 


\section{Les « obstacles épistémologiques » (G. Bachelard)}

« On ne connaît que contre la connaissance antérieure. »

G. Bachelard.

La nature, l'environnement, le paysage sont des entités familières qui ne se laissent pas facilement apprivoiser, pas plus dans le quotidien que dans la recherche, polysémiques sans pour autant être polyphoniques. D'où la difficulté à les aborder de front dans une perspective de comparaison et de rapprochement. Sur le sujet, la bibliographie générale est, certes, foisonnante. Mais si elle ouvre de larges horizons, elle ne propose pas beaucoup de pistes conceptuelles et encore moins méthodologiques et pratiques. Nous avons délibérément choisi de partir de notre pratique du terrain, de terrains divers où ces différentes problématiques paysagères et environnementales se rencontrent et convergent tout «naturellement». La Liebana (montagnes cantabriques, Espagne), le Sidobre (Sud-ouest du Massif central français), le Pantanal (Mato Grosso, Brésil) sont des entités géographiques variées qui nous ont permis d'avancer sans logomachies ni rhétoriques préalables. Cette pratique en rase-mottes ne s'écarte pourtant jamais des grandes théories et problématiques mais elle les ramène toujours à des questions de concept et de méthode appliqués. Les principes de cette «épistémologie de terrain » ont déjà été présentés $(\mathrm{C}$. et G. Bertrand, 2002 et 2014). Il s'agit de raisonner les difficultés concrètes ou théoriques rencontrées sur le terrain en termes d' « obstacles épistémologiques » (en s'inspirant du sens bachelardien). Autant de « seuils » qu'il faut d'abord reconnaître pour, soit les surmonter, soit les contourner... avec les moyens du bord. Cette priorité accordée aux faits du terrain se dégage de tout a priori disciplinaire ou interdisciplinaire en prenant cependant en compte l'ensemble des études scientifiques ou littéraires. Nous n'évoquerons ici que les principaux « obstacles épistémologiques » rencontrés. Ils conditionnent notre recherche sur la place et les fonctions à accorder à la nature. Souvent ils nous embarrassent, parfois ils nous bloquent, toujours ils nous stimulent.

\section{L'insurmontable diacrisis Nature-Culture}

Du point de vue épistémologique, c'est le premier obstacle. Il conditionne tous les autres. Fondement de la pensée occidentale, grecque et judéo-chrétienne, il borne deux champs philosophiques, voire métaphysiques et théologiques, considérés comme irréductibles. La division positiviste du travail scientifique entre sciences de la nature et sciences de la société l'entérine et la renforce... tout en l'occultant dans la pratique quotidienne de la recherche. De plus, la question a été dévoyée par l'utilisation courante du rapport bancal NatureSociété, comme si la société et les individus qui la composent n'avaient aucune existence biophysique. Max Sorre avait déjà souligné cette absurdité encore très répandue aujourd'hui. La plupart des disciplines évitent d'aborder la question et font comme si le rapport nature-culture allait de soit dans le cadre frileux 
de leurs problématiques. Les ethno-anthropologues ont traité directement du problème avec de nouveaux éclairages, en particulier à partir des mythes $(\mathrm{C}$. Lévi-Strauss, P. Perez) ou du concept transversal d'animalité (Ph. Descola). Il ne s'agit pas de vouloir surmonter l'insurmontable mais de prendre acte des interactions, interfaces et insterstices. L'obstacle est toujours devant nous. Il conditionne notre recherche.

\section{Les confusions autour du néo-déterminisme}

Le débat déterminisme-possibilisme dure toujours. Il vient d'être réactivé, sous la poussée théologico-mystique du créationnisme anti-darwinien et plus ou moins directement prolongé, sans trop de précautions philosophiques et scientifiques, par la relance aussi tardive que maladroite de l' " environnement history » américaine (G. Quenet). Si l'inconsistant possibilisme relève de l'art de l'esquive, le néo-déterminisme fausse la question de l'environnement et encourage toutes sortes d'élucubrations antirationnelles et catastrophistes. D'une façon générale l'ignorance, consciente ou inconsciente, des lois physiques et des fonctionnements biologiques en est une grave inconséquence que l'on retrouve dans beaucoup de recherches environnementales et, à plus forte raison, paysagères.

\section{Le couple infernal environnement-paysage}

Si l'expression choisie n'est pas très scientifique la situation ne l'est pas davantage. L'environnement et le paysage vivent une cohabitation compliquée faite d'emprunts, de collusions et de rejets. Ces deux grandes filières historiques, l'une d'inspiration naturelle sous-tendue par l'écologie, l'autre culturelle et artistique plus ou moins confortée par l'artialisation, n'arrivent pas à concevoir, sinon un objet, du moins un objectif commun. En France, deux ministères (l'Environnement et la Culture), sinon trois (l'Agriculture) se disputent autant l'environnement que le paysage sans trop s'occuper d'une naturalité qui les fonde, qui est partout et nulle part. En dépit des rafales de réformes universitaires, les formations pédagogiques restent dans un flou concurrentiel avec des issues professionnelles incertaines. Des logiques de rapprochement, comme celle de l'École de Paysage de Bordeaux, sous l'impulsion de S. Briffaud, ne sont pas toujours comprises et acceptées par le corporatisme ambiant. Il ne s'agit pas de tout fondre et confondre mais de trouver une voie de conciliation pour retrouver cette naturalité qui nous entoure et, pour partie, nous conditionne.

\section{Une diversité naturelle au-delà de la seule biodiversité}

La prise en compte de la biodiversité est une étape décisive dans l'approche scientifique de la nature, de son état actuel et de son évolution vers des dégradations et des appauvrissements de plus en plus irréversibles. Urgente, elle 
n'est cependant pas suffisante et ne doit pas masquer la relation globale culturenature.

D'une part la biodiversité, pour importante qu'elle soit comme source de vie et d'équilibre de la biosphère, n'est qu'un aspect de la diversité de la nature. Les autres éléments naturels, la roche, l'eau, l'air et le feu, ne peuvent être négligés ou seulement traités à travers leurs conséquences sur la biodiversité. Nous retiendrons tout particulièrement l'oubli fréquent de la diversité des modelés et des formes du relief qui, à toutes les échelles de temps et d'espace, participent à la formation des paysages et des environnements.

D'autre part, cette diversité naturelle ne peut être séparée de la diversité des sociétés et de leurs cultures sans lesquelles les paysages et l'environnement, mais aussi la nature, sont incompréhensibles. Par exemple, la relation entre biodiversité et paysage est loin d'être correctement traitée.

\section{Ni discipline, ni interdiscipline : éloge de l'indisciplinarité, vers une recomposition des savoirs}

Poser simplement la question de la nature est suffisamment incongru pour ébranler quelques certitudes académiques autant disciplinaires qu'interdisciplinaires. Les problématiques sont en porte-à-faux, trop sectorielles, trop scientistes, pas assez culturelles et, de plus, trop divergentes pour être efficaces. Les nombreuses tentatives interdisciplinaires sont le plus souvent restées à l'état d'intentions théoriques ou bien n'ont produit que des « copier-coller » disciplinaires dans le cadre de monographies convenues. Par exemple, évoquer la co-évolution nature-société est un aveu d'échec, l'addition de parties séparées ne peut pas déboucher sur le tout.

Des chercheurs conscients de la nécessité d'innover se sont plus ou moins directement tournés vers les pratiques de gestion et aménagement des territoires qui sont de plus en plus en prise avec les questions de nature, d'environnement, de paysage. Les recherches de «paysage-action » en sont un bon exemple. C'est parmi ces tentatives dispersées mais scientifiquement efficaces qu'il faut, au moins dans un premier temps, rechercher, sinon des méthodes innovantes, du moins des « biais » significatifs (J.-P. Deffontaines, Y. Michelin, L. Lelli, G. Domon). Nous faisons nôtre, dans un tout autre contexte scientifique, le plaidoyer du biométricien J.-M. Legay en faveur d'une indisciplinarité de principe dans la perspective d'une recomposition des savoirs. Le concept de nature-artefact constitue pour nous une première étape. 


\section{- La nature-artefact}

« Réinventer une nature contemporaine, incompréhensible sans les hommes comme ceux-ci sont incompréhensibles sans la nature, creuset des origines, souffle de vie, paysage, patrimoine. » Armand Frémont.

Il y a chez Armand Frémont cette vision généreuse, à la fois humble et ambitieuse, qui tranche parmi les tirades trop souvent convenues et présomptueuses de ceux qui font profession de penser la nature. C'est celle d'un géographe resté, au plus près des hommes, à l'écoute des mille bruissements du bocage normand et de la forêt d'Ecouves.

« Réinventer la nature » est bien autre chose qu'un projet scientifique. C'est un projet de vie. Une ligne de fuite vers un inaccessible horizon ; une utopie pourtant nécessaire pour rassembler la fragmentation contemporaine des cultures et des savoirs, leur confinement dans des boîtes à outils aux compartiments de plus en plus étroits et étanches, sans échappée sur la complexité-diversité et sur la beauté du monde.

Depuis toujours nous nous interrogeons sur la nature. Moins la nature majuscule et transcendantale que les diverses natures rencontrées au travers de paysages familiers ou lointains, ordinaires ou extraordinaires, réels ou imaginaires. Obstacle après obstacle nous avons composé un protocole d'analyse générale, le système GTP (Géosystème-Territoire-Paysage). Il tente de prendre en compte le milieu géographique au sens large, à la fois physique et culturel. Cette unité territoriale ne doit pas masquer la diversité interne du système dans lequel sont ménagés trois entrées et trois sous-systèmes qui sont autant de filières :

- le Géosystème, entrée et sous-système naturaliste anthropisé ;

- le Territoire, entrée et sous-système socio-économique ;

- le Paysage, entrée sous-système socio-culturel.

La nature y est partout présente sous différentes compositions et perspectives, toujours métissée ; à la fois source, ressource et ressourcement.

\section{Les fondements d'un concept oxymore}

\section{Le double artefact anthropisation-artialisation}

L'artificialisation est la pierre angulaire de la méthode. Elle fait éclater les catégories conceptuelles et les recompose. L'artificialisation, certains utilisent le terme moins fréquent d'artéfaction, est prise ici dans son sens le plus commun et le plus large. L'artus factum est le produit, à partir de la matérialité de la nature, de l'activité humaine dans sa globalité indissociable à la fois idéelle et matérielle. Qu'elle soit créatrice ou destructrice, c'est-à-dire sans $a$ priori et jugement de valeur. Cela revient à rapprocher, sans les fondre, les deux formes de l'emprise humaine sur la nature qu'une pensée analytique et 
positiviste a depuis longtemps séparées et éloignées aux deux extrémités du champ scientifique :

- L'anthropisation, au sens large d'impact matériel sur la nature, dont l'une des premières manifestations prise scientifiquement en compte a été l'érosion des sols consécutive aux défrichements. Dans notre définition il s'agit de l'ensemble des activités matérielles des sociétés... au moins depuis le néolithique en Europe (agricoles, industrielles, urbaines).

- L'artialisation, au sens large de l'ensemble de la transformationtransfiguration de la nature par toutes les formes de représentation de la pensée et de la sensibilité humaines, des arts, des émotions et des rêves. Une métamorphose de la nature. Cette définition constitue un considérable élargissement par rapport au sens habituel des paysagistes qui limitent l'artialisation à la peinture et aux jardins.

Ce rapprochement constitue la première étape dans l'élaboration de la méthode :

- C'est la reconnaissance a priori du mélange et de l'artifice que constitue la nature au travers des paysages, des environnements, des territoires. Il n'y a là rien de bien nouveau. Toutefois, cette affirmation devrait nous contraindre à ne plus parler de la Nature comme d'une entité à part entière et opposable à la Société. Dans notre proposition il n'y a pas de nature naturelle ; il y a seulement des natures dans les cultures ; les sociétés sont des entre-deux.

- A contrario, nous considérons que cette nature artificialisée existe en ellemême et pour elle-même dans sa matérialité biophysique de roche et d'eau, d'air et de feu, d'animaux et de végétaux. Un socle irréductible de naturalité sur lequel s'exerce une artificialisation sous toutes ses formes et dans toute sa dimension historique. Une conception inversée par rapport aux notions classiques d'environnement et de paysage où la nature est subordonnée, domestiquée et masquée par l'emprise humaine.

Le concept métissé de nature-artefact a été façonné, à la longue, comme une nécessité de terrain. L'arbre du bocage est bien à la fois du naturel et de l'artifice. Cependant et de toute évidence, cette conception banale s'appuie sur une longue tradition philosophique et scientifique. Nous n'en rappellerons ici que les manifestations les plus récentes qui ont directement influencé notre proposition :

- En premier lieu, le plaidoyer utopique et enthousiasmant, malheureusement peu connu, de l'historien J. Chesneaux qui a imaginé une virtuelle « collision nature-culture » comme perspective d'avenir (Barrué et Bertrand, 2000).

- L'analyse scientifique pour « un temps du mélange » du physicien E. Guyon (Barrué et Bertrand, 2000).

- Les diverses propositions d'E. Morin concernant la mise en place d'un paradigme de la complexité-diversité. 
- L'esquisse d'une «pensée métisse » proposée en particulier par I. Stengers, qui séduit par sa liberté sémantique et son ouverture épistémologique, sans pour autant constituer encore une référence méthodologique généralisable (Repères prospectifs, 2003).

Le métissage et la double artificialisation de la nature-artefact ne seraient que des pétitions de principe si elles ne bousculaient pas certains fondements des études environnementales et paysagères, parmi lesquels l'incontournable « déterminisme naturel » et bien d'autres considérations coupées de leur double origine culturelle et naturelle.

L'enjeu du « déterminisme naturel » dans le jeu des déterminismes sociaux

L'explicitation du concept de nature-artefact nous conduit à traiter la question récurrente et embarrassante du « déterminisme naturel » et de son corollaire le possibilisme, sans que nous possédions une réponse philosophique d'ensemble, tout au plus quelques principes pour avancer au cas par cas et qu'il serait hasardeux de généraliser sous une formulation univoque et définitive.

Le fait de traiter directement de la nature c'est lui reconnaître un rôle certain, sinon globalement déterminant du moins dans certaines conditions bien déterminées, en fonction des lois physiques et des fonctionnements biologiques universels. "L'eau de la rivière coule dans toutes les rivières au même temps » (J. et J.M.G. Le Clézio).

Dans l'Histoire de la France rurale (1970), nous avions évoqué l'existence de « seuils agro-techniques » tels que le remplacement de l'araire par la charrue à versoir ou bien l'introduction de la luzerne dans l'assolement; ce qui ne modifie en rien la nature de la nature mais en révolutionne l'usage et en surmonte le déterminisme. Aujourd'hui, l'explication paraît un peu courte, à l'exemple de la «fertilité sociale » des sols telle que la définissent les agronomes (J.-P. Deffontaines). Ce filtrage culturel de la détermination naturelle est magistralement décrit par P. Perez à propos de l'invention chamanique de leurs paysages-territoires par les indiens Hopi (Arizona). Dans d'autres conditions d'artialisation culturelle, socio-économique et technologique, la détermination naturelle joue à plein, par exemple pour l'agriculteur gersois qui surveille son champ de blé ou pour le citadin toulousain qui soigne son pot de fleurs. À chacun sa nature ! À chacun son déterminisme !

Cela signifie qu'il est philosophiquement irrationnel et scientifiquement erroné de vouloir traiter d'un déterminisme naturel généralisé sans le replacer dans la complexité-diversité des multiples déterminismes sociaux, immatériels ou matériels, qui pèsent lourdement sur les sociétés mais très différemment selon les économies, les classes ou catégories sociales ou les individus. Au rythme d'une même saison, les déterminants ne sont pas les mêmes pour l'éleveur et le viticulteur voisin, l'un attend la pluie, l'autre le soleil. Dans ces conditions la 
« ressource naturelle » et la « contrainte » ne sont jamais pleinement naturelles mais les « inventions » d'une société pour une activité donnée dans un temps donné.

L'édifice ainsi construit est un empilement de déterminismes naturels et sociaux, partiels et partiaux, souvent contradictoires, qui évoluent dans des sens et avec des rythmes différents. Le concept de nature-artefact participe de ce feuilletage déterministe qui ne peut pas être expliqué dans le cadre d'un possibilisme évanescent, même remis au goût du jour (V. Berdoulay). Demeure le jeu des déterminismes. En plagiant G. Perec nous dirons que la vie consiste à passer d'un déterminisme à l'autre en essayant de ne pas se cogner. On se cogne souvent à la nature.

\section{La mise en horizon du territoire}

Tout commence par le regard. Sur le terrain il scrute et balaie, délimite et détaille, séparant les plans, sautant de masques en échappées, jouant avec les ombres et les lumières, égrenant forêts et villages. Il hiérarchise, borne et élimine. Il transmue le territoire en paysage, l'artificialise et l'artialise. Il le poétise aussi. La nature-artefact naît dans les replis toujours mystérieux du paysage, avec une part incompressible d'émotion et de rêve.

Cette étape de la représentation paysagère est fondatrice du concept de natureartefact dans la mesure où elle saisit la nature au travers d'un filtrage culturel, à hauteur d'homme, qu'elle la « verticalise » et par là l'humanise. La nature n'est plus cette entité neutre et froide qui surplombe ou côtoie la société. La nature-artefact a non seulement un horizon qui l'individualise, mais de multiples horizons à découvrir. Une vision à la fois panoramique et cinématique dans la mesure où la nature-artefact s'inscrit dans la mobilité générale de la société. La vitesse, et son accélération, sont des données essentielles de l'analyse de la nature-artefact. Par exemple, au sein de cette mobilité généralisée il y a le ciel, ce grand oublié des analyses naturalistes et paysagères. Comment parcourir le Sertão brésilien assoiffé et sa misère agraire sans suivre des yeux les trains de petits nuages cotonneux qui filent dans le courant de l'alizé mais qui ne versent jamais d'eau ? La nature-artefact est bien le lieu « où la terre et le ciel se rencontrent », ce n'est pas seulement un paysage à regarder mais une terre à vivre, ou à fuir... vers d'autres horizons.

De la discordance des temporalités à la " tresse des temps » (D. Guillemet)

«C'est le geste du temps qui gobera tous les autres. »

Rosando $\mathrm{Li}$, poète péruvien.

L'émiettement des temporalités et l'enchevêtrement des chronologies compliquent l'histoire générale de l'environnement, tout particulièrement entre l'histoire de la nature et l'histoire de la société. De plus, les interactions temporospatiales n'échappent pas toujours à des explications mécanistes. Le colloque 
du PIREVS-CNRS (Géode, Toulouse, 1997) sur « les temps de l'environnement » et l'article sur « la discordance des temps » (CNRS, Besançon, 2002) soulignent le paradoxe des recherches sur le temps. Une question centrale mais le plus souvent réduite à la seule chronologie.

Le concept de nature-artefact pose très directement la question d'un espacetemps sinon unifié, du moins rassemblé autour d'une certaine « concordance des temps » : entre nature et société, entre le temps qui passe (time) et le temps qu'il fait (weather), entre le temps rond des saisons (phénologie) et le temps long, en replaçant les crises naturelles et les catastrophes humaines dans la longue durée du climat.

Le concept de nature-artefact nous amène à reconsidérer beaucoup d'autres questions vives qui sont généralement abordées isolément, sans rapport avec la culture et la nature : par exemple un discours sur la durabilité dans un espacetemps non défini ou bien des références de principe à la renouvelabilité dans l'irréversibilité de la flèche du temps. Des tergiversations et incertitudes scientifiques laissent libre cours à des élucubrations apocalyptiques et polluent le discours scientifique contemporain, tout particulièrement celui sur le changement climatique.

\section{L'artificialisation (artéfaction) « projet » central du système GTP}

La nature-artefact participe d'une recherche méthodologique au long cours consacrée au rapport nature-culture. La première étape a été, pendant la décennie 1960, l'élaboration du concept et de la méthode du géosystème. Concept de finalité naturaliste tout en prenant en compte l'anthropisation des milieux. Aujourd'hui, le concept de nature-artefact a été conçu et mis en pratique dans le cadre épistémologique et méthodologique plus large du GTP. Il en constitue une avancée théorique, tout particulièrement en mettant la nature en scène dans la culture.

\section{L'artificialisation comme principe téléologique du GTP}

Le GTP a été, dès l'origine (1990-2000), conçu comme une configuration systémique embrassant l'ensemble du complexe territorial, environnemental et paysage. Comme toute analyse de système, le GTP est organisé autour d'un principe téléologique. Il est autocentré et autosuffisant. Toutefois des expériences conduites sur plusieurs terrains avec des problématiques différentes ont démontré que cette finalité générale du GTP était trop exhaustive et théorique et que le projet de recherche n'évitait pas un certain flottement conceptuel. Comme toute analyse de système le GTP doit être fléché, avec une finalité univoque et extérieure à son propre fonctionnement. Le choix de l'artificialisation comme principe téléologique du GTP s'est imposé de lui-même comme réponse directe et globale à la crise générale contemporaine de la planète ; à l'interface 
des questions de nature et de culture ; au cœur d'une vaste problématique dont on ne maittrise pas tous les aspects. La nature-artefact jouant le rôle opératoire de concept central et centralisateur.

Nous ne sommes pas encore à même d'explorer toutes les possibilités épistémologiques et méthodologiques offertes par ce dispositif. Cependant il est déjà possible d'évoquer quelques perspectives et de préfigurer quelques axes de recherche :

- Le métissage des temporalités. L'artificialisation donne non seulement du sens mais aussi un sens à l'histoire de l'environnement qui n'est ni l'histoire de l'écologie et de l'écosystème, ni l'histoire humaine du «temps des horloges » (J. Le Goff). Les interprétations fixistes ou strictement cycliques (climax) sont à revoir ainsi que l'ensemble des processus temporels tels que la réversibilité et la renouvelabilité.

- La coordination entre paysagisme et environnementalisme. Les expertises sectorielles, soit paysagères, soit environnementales, peuvent conduire à de lourdes aberrations technocratiques et politiques. Par exemple quand on classe internationalement de grands vignobles en fonction de critères paysagers, esthétiques, patrimoniaux et identitaires en faisant semblant d'ignorer l'accumulation irréversible de pesticides et autres polluants ainsi que le développement de maladies professionnelles, certaines mortelles.

- L' « invasion » n'est pas la «peste ». Le concept de nature-artefact nous amène à revoir les notions de mobilité, dispersion, adaptation des êtres vivants. Toutes les espèces dites « invasives » ne sont pas des pestes. Que seraient sans elles notre agriculture, nos espaces ruraux et notre alimentation ? La nature-artefact participe aussi d'un combat idéologique qui s'appuie sur l'histoire longue de tous les mélanges et métissages.

- Les «états » du GTP et les trajectoires d'artificialisation. Au plan de la méthode la combinaison culture-nature permet non seulement de mieux définir l'état global du GTP (comme c'est le cas pour le géosystème), mais encore de définir des trajectoires d'artificialisation et peut-être, un jour, de savoir les prolonger.

\section{La consolidation de la méthode GTP}

Le principe téléologique de la nature-artefact fondé sur sa double artificialisation donne au système GTP une unité conceptuelle et une vigueur méthodologique qu'il n'avait pas totalement acquise sur le plan théorique et qui ne facilitait pas sa mise en pratique. L'artificialisation constitue un fil rouge, sinon exclusif, du moins privilégié. Sans remettre en question la dimension systémique sur laquelle repose la méthode du GTP, elle l'infléchit et la complète. 
- Le recours à une monographie systémique territorialisée. Non sans raison mais non sans excès on a tous critiqué la monographie géographique classique, ses tiroirs bien rangés, ses descriptions convenues et sa finalité incertaine, sans pour autant cesser de la pratiquer, sans l'améliorer ni proposer d'autres protocoles scientifiques. La définition traditionnelle n'est pas acceptable : « étude complète et détaillée qui se propose d'étudier un sujet précis relativement restreint» (Petit Robert). Des sociologues (M. Jollivet) ont introduit des réajustements pour dynamiser et ouvrir la monographie. Le GTP peut être considéré comme une construction monographique territorialisée et systémisée. L'artificialisation lui fournit la dimension téléologique qui lui fait défaut. Cette hypothèse, déjà testée à l'occasion des Rencontres scientifiques d'Arthous, a permis de construire une interprétation d'ensemble du Pays d'Orthe (Landes) combinant le paysage à l'environnement, le culturel au naturel, les « barthes » inondables de l'Adour à l'abbaye bénédictine de Sorde. En donnant existence au Pays d'Orthe dans sa diversité géographique et sa profondeur historique (G. Bertrand et S. Briffaud, 2008, tout particulièrement l'étude de terrain de R. Bercovitz, A. Luginbühl, A. Rol-Tanguy).

- Le GTP mis en scène. Le concept de nature-artefact, au travers de l'artialisation, confère au GTP une dimension sensible et esthétique qui transfigure sa matérialité. C'est la dimension proprement paysagère du GTP. Une des manifestations la plus évidente est l'appréhension panoramique et la mise en horizon du territoire. Le panorama revient ainsi au centre de la méthode, avec toutes les techniques appropriées (descriptif oral et écrit, croquis, photographies, etc.). C'est une belle leçon pratique de terrain donnée par les paysagistes que les géographes ont un peu trop tendance à négliger. Le GTP relève donc d'une mise en scène scientifiquement construite et « artialement » mise en scène. La méthode du GTP se prolonge par des essais de scénographes qui testent sur le terrain quelques principes de mise en scène empruntés au théâtre le plus classique, en particulier la fameuse règle des trois unités qu'il n'est même pas nécessaire de dépoussiérer. En effet, la monographie systémisée et territorialisée du GTP s'accorde parfaitement avec l'unité d'action, de temps et de lieu. À condition de jouer sur les échelles temporo-spatiales internes et externes à la monographie elle-même. Carrières granitières et plantations résineuses du Sidobre, pelouses pastorales des puertos cantabriques, rivières du Pantanal polluées par des garimperos, soulanes ariégeoises, pignadars landais, hauts versants terrassés de la vallée du Khumbu (Népal) participent de la nature-artefact, de son décor et de son spectacle. L'Anthropocène muée en anthropo(s)cènes !

La nature-artefact nous fait entrer dans l'Anthropocène par la grande porte. Celle de l'artificialisation irréversible de la nature qui s'accélère avec toutes les misères et les beautés du monde. Cette artialisation-anthropisation marque 
paradoxalement un retour en force de la nature avec des émerveillements toujours renouvelés et des interrogations de plus en plus inquiètes. Sous l'artefact... la nature.

\section{Vibliographie}

Arcy F., Bruston A., Maffesoli M., Poche B., Baudrillard J., 1977, État de la nature et nature de l'État, Université des Sciences Sociales de Grenoble.

Barrué M. et Bertrand G (sous la direction), 2000, Les temps de l'environnement, Colloque CNRS, Presses universitaires du Mirail.

Berdoulay V., 1988, Des mots et des lieux. La dynamique du discours géographique, Paris, CNRS, « Mémoires et Documents».

Berque A., 2000, Écoumène. Introduction à l'étude des milieux humains, Paris, Belin.

Bertrand C. et G., 2002, Une géographie traversière. L'environnement à travers territoires et temporalités, Paris, Arguments.

Bertrand C et G, 2014, Du terrain au concept. Une expérience d'« épistémologie de terrain », Université de Santander (sous presse).

Bertrand C. et G., 2014, Projet de paysage ou projet de territoire ?, Toulouse, Sud-Ouest européen(à paraître).

Bertrand G. et Briffaud S. (dir.), 2008, Le paysage. Retour d'expériences entre recherche et projet, Actes des Rencontres d'Arthous, conseil général des Landes.

Briffaud S., 2014, « Le paysage, le regard et le temps. Enquête historique et géographique sur l'empaysagement des espaces et des sociétés », Texte de soutenance HDR, Université de Bordeaux III (en cours de publication).

Deffontaines J.-P., 1998, Les sentiers d'un géoagronome, Paris, Arguments.

Descola Ph., 1986, La nature domestique, Paris, Maison des Sciences de l'Homme.

Domon G. (dir.), 2009, Le paysage humanisé au Québec, Presses Universitaires de Montréal.

Dubost F. et Lizet B., 2003, "La nature dans la cité », in Bienfaisante nature, Paris, Seuil.

Frémont A., 2012, Normandie sensible, Paris, Éditions Cercle d'Art.

Galop D., 1998, La forêt, l'homme et le troupeau dans les Pyrénées, Université de Toulouse-Le Mirail.

Guille-Escuret G., 1984, Le fait social contre nature, Paris, Les Temps Modernes $n^{\circ} 451$.

Legay J.-M., 1997, L'expérience et le modèle, Paris, INRA éditions, Sciences en question.

Lelli L., Sahuc Ph., 2004, Le paysage, une interface de dialogue entre chercheurs et acteurs, Agricultures et paysage (sous la direction de G. Domon et J. Ruiz), Presses Universitaires de Montréal.

Métailié J.-P. et Bertrand G., 2006, Les mots de l'environnement, Toulouse, Presses universitaires du Mirail.

Moscovici S., 1968, Essai sur l'histoire humaine de la nature, Paris, Flammarion.

Perez P., 1998, Le monde au-delà du bambou, Thèse de doctorat, Paris, EHESS.

Quenet G., 2014, Qu'est-ce que l'histoire de l'environnement ?, Paris, Champvallon. 
Repères prospectifs (collectif), 2003, La pensée métisse $n^{\circ} 100$, Paris, ministère de l'Équipement. Roger A., 1997, Court traité du paysage, Paris, NRF Gallimard.

Stengers I., 2003, "Et si un jour les sciences devenaient civilisées », Repères prospectifs (collectif), Paris, ministère de l'Équipement.

Walter F., 2014, Paysage et environnement en histoire. Échapper au brouillage, L'Information géographique, Paris (dans ce même numéro). 